\title{
Using Rendezvous to Overcome Communication Limitations in Multirobot Exploration
}

\author{
Briana Lowe Wellman*, Shameka Dawson*, Julian de Hoog ${ }^{\dagger}$, and Monica Anderson* \\ *Department of Computer Science \\ The University of Alabama, Tuscaloosa, AL, US \\ Email: blowe, sdawson, anderson@cs.ua.edu \\ $\dagger$ Department of Computer Science \\ University of Oxford, Oxford OX13QD, UK \\ Email: julian.dehoog@cs.ox.ac.uk
}

\begin{abstract}
In cooperative multirobot systems, communication can speed up completion, reduce redundancy, and prevent interference between robots. Typically, wireless point-to-point communication is used to coordinate robots. However, environmental interference, unpredictable network conditions, and distances between robots can affect the reliability of wireless communication. Therefore, approaches other than continuous message passing throughout exploration are useful.

We consider the problem of coordinating a multirobot system to explore an unknown, large, open environment. An approach that uses sector search with rendezvous is presented. Robots explore an environment in sectors, or designated areas, and periodically meet to communicate map information of what they have explored. Our approach is compared to other communication paradigms in simulation. Results suggest that sector search with rendezvous is more efficient than having no communications. It further demonstrates advantages over scenarios in which robots communicate only with other robots in close proximity, and is comparable to a role-based approach with dynamic team hierarchies.
\end{abstract}

Index Terms-Multiagent Systems, Distributed Systems, Cooperation, Communication

\section{INTRODUCTION}

Cooperative multirobot systems are advantageous in hazardous coverage applications such as search and rescue, surveillance, and toxic waste clean-up. Deploying robots instead of humans can prevent human casualties as well as speed up completion. To cooperatively complete tasks so that work is not duplicated, robots can cooperate by sharing digital information. A messaging capability can prevent robots from interfering with one another and reduce duplication in coverage [1]. However, intra-team digital communications are not always guaranteed as communication can often be unreliable.

Since many hazardous applications require coverage of large unknown environments, wireless ad-hoc networks are often utilized. Typically, wireless networks can be rapidly deployed due to minimal configuration requirements. However, communication performance is affected by environmental interference (e.g. walls) and decreases as the distance between robots increases [2]. In addition, if robots exchange large amounts of information then they run into the risk of receiving incomplete information due to cpu overload from individual message processing [3]. As message loads increase, team performance degrades. To address these challenges, alternative approaches to continuous message passing are useful.

Approaches that do not use any message passing to coordinate robots include: potential fields [4], [5], [6] where robots attract and repel each other, and ant or swarm robots [7], [8] where virtual pheromones or trail markings are used to communicate and influence robot behavior. However, both approaches depend on local interactions where after a certain distance they no longer coordinate.

Other researchers that consider message passing with network constraints include: [9], [10] in which robots are required to maintain line-of-sight with other robots, [11] in which message size is reduced by allowing robots to communicate polygonal representations of the map, and [12] where rendezvous approaches allow robots to meet up to exchange information about the environment.

In this paper, an approach using sector search with rendezvous to coordinate a robot team in an unknown environment is presented. Instead of continuously passing messages throughout the entire exploration, robots explore their sector and then rendezvous to communicate what was found. We use small teams of intelligent robots to cover a large open area that represents an outdoor environment such as a city block. We compare our approach to other direct communication and rendezvous approaches. We hypothesize that sector search with rendezvous is efficient in coordinating robots in coverage tasks of wide open areas.

In Section II, related work is presented. A discussion of our approach follows in Section III. In Sections IV and V, simulation set up and results are discussed. An analysis of the results is provided in Section VI. Finally, Section VII presents conclusions and future work.

\section{RELATED WORKS}

Several researchers have proposed approaches for coordinating robot teams without message passing.

One approach involves coordinating robots with potential fields. In [4], [5], Howard et al. present a distributed virtual field force where robots are subject to repulsion from other robots and obstacles, causing robots to spread out. While this approach depends on local interactions which can only 
occur when robots are in close proximity to one another, our approach allows robots to use interaction information even after robots can no longer sense each other.

Other approaches have been implemented with consideration of network constraints. Several researchers have looked into requiring robots to maintain line-of-sight so that robots remain in communication range with each other. Rekleitis et al. [10] address the implementation of coordinating robots when information sharing is restricted to line-of-sight communication in an unknown environment. Their results show that maintaining the cohesiveness of the team by allowing only minimal splitting leads to a reduction in repeat coverage.

In similar work, Arkin et al. [9] investigate how a team of robots can self-organize during exploration by maintaining line-of-sight communications. Experiments involve robots searching for hazardous materials with varying degrees of prior knowledge. The line-of-sight approaches work well when there is a requirement for robot cohesiveness, but in general will not be as efficient in a large environment when a small number of robots have to spread out more to cover the environment.

Senthilkumar and Bharadwaj [13] present an approach to the robot path exploration problem that allows robots to find a collision free path to reach predetermined points in an environment. They use a complete weighted graph that represents the points in the environment which the robots have to explore using a shortest path. They divide the area into clusters and assign each robot a cluster to explore. After visiting all points in the cluster using a Traveling Salesman Problem approach, the robots meet at the predefined rendezvous point. However, they make off-line decisions which require the topology to be known in advance, limiting the ability to react to dynamic environments.

In [11], Meier et al. present a technique for assigning targets to robots and deciding what information to transmit when using communication with limited bandwidth. Each robot explores an unknown environment and creates a polygonal approximation of a map. To reduce message sizes, polygonal representations of the map are communicated. Using this approach, Meier et al. were able to effectively coordinate a team of robots under bandwidth limitations. Nevertheless, communicating polygonal representations still requires that network connectivity exists.

Many researchers have presented work on using rendezvous techniques. One approach is demonstrated by Roy and Dudek in [12]. The focus is on getting robots to meet at a rendezvous location if they do not know each other's initial start positions. The goal is to explore the environment and meet at a location with robots that have noisy sensors.

Another approach using rendezvous in an unknown environment is presented in [14]. De Hoog et al. use role-based exploration where some robots (Relays) relay information between robots and a central command centre while others (Explorers) continue to explore using frontier exploration. When a Relay and Explorer meet, they share information about the environment and the Explorer plans the next rendezvous point. The Explorer determines the next rendezvous point by placing it deep into its next choice of frontier while ensuring that point has strong communication range. In [15], de Hoog et al. further develop this approach by proposing a dynamic team hierarchy in which robots swap roles to shorten paths for faster exploration.

\section{APPROACH}

In [16], Balch and Arkin found that the most complex communication schemes are not always necessary in tasks in which lower level types of communication, such as implicit communication, are effective. More complex communications do not always speed up completion. When message loads increase, team performance can degrade because robots are spending time processing messages instead of exploring.

In our approach, robots do not communicate during the entire exploration. Instead, robots are well coordinated using sector search so they are able to explore different areas without communicating. They use scheduled rendezvous to communicate new information. In the following sections, sector search and rendezvous are discussed.

\section{A. Frontier-based Exploration}

All approaches in this research use the coverage algorithm, frontier-based exploration. In a frontier-based [17] algorithm, robots recursively explore an unknown area while building a cellular representation, i.e. an occupancy grid [18]. Robots use a distance sensor to sense the space around them, allowing them to label each cell open, occupied, frontier, or unknown. Frontier cells are those cells on the borders between open and unknown cells.

When the robots communicate, they explicitly broadcast messages to all other robots. These messages are deliberately transmitted and received through point to point communications. Information about open cells is shared.

\section{B. Sector Search with Rendezvous}

The Sector Search with Rendezvous approach is designed to allow a robot team to explore an unknown environment. The goal of the robots is to collaboratively explore a large open area without using continuous message passing throughout the entire exploration. Instead, robots rendezvous to exchange information about what they have discovered. The following assumptions are made:

- Robots are equipped with a global positioning sensor (GPS) for localization and share a global map.

- The environment is wide open and unknown (e.g. outdoor).

- Robots start close to each other and share a common frame of reference.

- Robots are homogeneous and capable of exploring the environment on their own.

- Robot teams are small allowing for a limited number of messages at rendezvous.

- When robots communicate, messages are intentionally transmitted and received from robot to robot. 

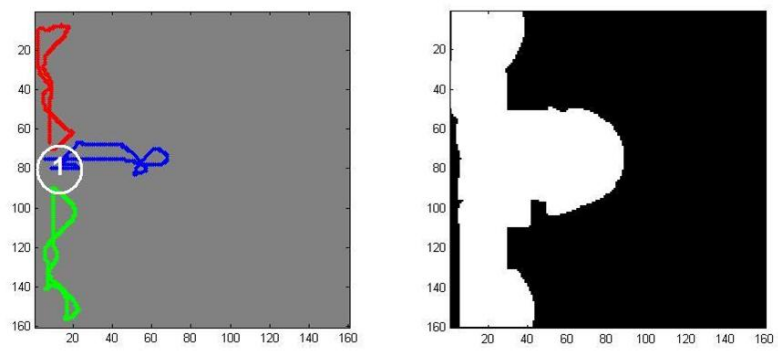

Fig. 1. The robots search in sectors, or designated areas, and then rendezvous to exchange map information. The paths of the robots are shown, with the starting point and initial rendezvous location at point 1 . The diagram on the right shows the area that has been covered.
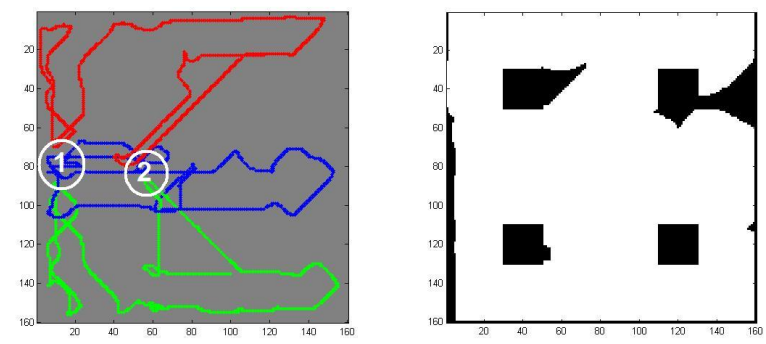

Fig. 2. The robots search in sectors, or designated areas, and then rendezvous to exchange map information. The paths of the robots are shown, with the starting point and initial rendezvous location at point 1.Subsequent rendezvous locations are determine after each meeting. The second rendezvous location is shown at point 2 . The diagram on the right shows the area that has been covered.

1) The Algorithm: While frontier-based exploration allows for robots to select frontier areas to explore based on individual utility, sector search allows robots to explore a pre-agreed coverage area. The area is divided into designated areas for each robot. The team decides on designated areas from similar start locations. For example, a robot may be designated to explore the most eastern part of the environment while another is designated to explore the most northern part. Because dividing the area into designated areas can cause uneven assignment, robots can explore outside their sectors after completing their own sector.

Although the robots are exploring designated areas within sectors, they still execute the frontier-based exploration algorithm within their area. Sector search is beneficial in large environments with few robots because robots spread out more in their assigned areas. If the environment is large and they do not explore in designated areas then they may end up exploring in the same area. In addition, when robots rendezvous, it is more likely that they are sharing new area information if they explore in designated areas.

2) Sector Designation: Although the environment is unknown, robots can be designated sectors. In the beginning of coverage, robots start at adjacent locations. This area of the environment can be represented as a semicircle. The arc of a semicircle always measures to $180^{\circ}$. If there are $N$ robots then $180^{\circ} / N$ indicates the angles of the sectors robots should cover. For example, in the experiments of this work, there are three robots. Therefore, robots distribute at $60^{\circ}$ angles from each other.

3) Rendezvous Locations: One of the most important factors in rendezvous is choosing rendezvous locations. It is difficult to determine locations in an unknown environment. Robots explore and build a map individually to share with other robots. Although, they share map information when they meet, they may not always have the complete map. For example, robots may not agree on a location because the location does not lie in every robot's map. In addition, robots may not make it to a location in time. In both cases, it results in robots having partial and varying maps.

In our algorithm, rendezvous locations are determined individually by each robot. The robots start exploration in adjacent locations. The initial rendezvous location is at this start point. Subsequent rendezvous locations are not decided on until after each rendezvous. If robots have successful rendezvous, they will have similar maps after meeting. On the contrary, if they do have successful rendezvous and are not able to communicate, then they will not have similar maps. However, after each scheduled rendezvous, robots choose the most central point in their map as the next rendezvous location.

Figures 2 and 3 illustrate the paths of a three-robot team exploring in sectors and their first two rendezvous locations. The location at point 1 shows the starting points of the robots and the initial rendezvous location. After the initial rendezvous, the robots each decide on their next rendezvous located at point 2.

4) Rendezvous Parameters: Other important aspects of rendezvous are choosing the time between when robots should rendezvous and the duration of the rendezvous itself. Having robots meet not often enough can result in a large amount of messages being passed when they do meet. However, if they meet too often, they spend more time meeting than exploring.

The duration of a rendezvous can also be influential on performance. If the duration is not long enough, then robots may not have enough time to make it to the rendezvous point and exchange information. In contrast, if the duration is too long, then they are wasting time after communicating. These two parameters are tested by two variations of the algorithm: SECTOR REND 1 and SECTOR REND 2.

In SECTOR REND 1, communication is limited in that only information that was discovered after the last rendezvous will be shared. In other words, environment information is shared only once at the next rendezvous. Therefore, if robots are not able to make it to the rendezvous location, they will miss the information sent by other robots. However, with SECTOR REND 2, robots send all the map information they have gathered from beginning of execution every time they meet. Therefore, SECTOR REND 2 should be assigned a longer rendezvous duration than SECTOR REND 1.

For both SECtor REND 1 and Sector Rend 2, the initial rendezvous is set to happen after two minutes of exploring. 
Subsequent rendezvous are every five minutes. This gives the robots enough time to cover a good portion of the environment and return. Sufficient rendezvous duration and meeting times can be determined by considering the robots' velocity (v), number of robots, and amount of communication at rendezvous. If there are $N$ robots and they meet every $t$ minutes then the amount of communication can be approximated by $N \times t \times v$. The rendezvous durations for SECTOR REND 1 and SECTOR REND 2 are two minutes and six minutes; respectively. Additional time is needed for SECTOR REND 2 since the robots communicate all environment information that was found from the beginning of the search every rendezvous.

\section{Approaches for Comparison}

To demonstrate sector search with rendezvous, it is compared to No Comm, Prox Comm, Full Comm, and the ROLE-BASED approach described in [15].

In the No COMM approach, there is no direct communications between robots. Each robot only relies on their perception of the environment. However, with the FULL COMM and PROX COMM approaches, robots explicitly broadcast messages to all other robots. The only difference between the FULL COMM and PROX COMM is that with PROX COMM robots only communicate with other robots that they sense within five meters. The following section describes in more detail the ROLE-BASED presented in [15].

\section{Role-based with Dynamic Team Hierarchy}

In [15], de Hoog et al. demonstrated role-based exploration with a dynamic team hierarchy. As noted in the related work section, some robots (Relays) relay information between robots and a central command centre while others (Explorers) continue to explore using frontier exploration. Subsequent rendezvous points are pushed deeper and deeper into the environment, leading to full exploration. When two robots enter one another's communication range, they examine whether it is advantageous for them to swap roles, i.e. for each to take on the other's task. If yes, they swap roles and exploration continues.

The main differences between sector-based exploration and role-based exploration are:

- role-based exploration requires a team hierarchy whereas sector-based exploration does not

- in role-based exploration, meetings occur between robots pairwise whereas in sector-based exploration all robots meet simultaneously

- role-based exploration typically aims to gather all information at a single, central location, which is not the case in sector-based exploration

\section{Simulation Setup}

The Sector Rend, No Comm, Full Comm and Prox Comm approaches were simulated in the 3-D physics-based simulator, Webots [19]. In Webots, a wheel encoder noise (based on a Gaussian distribution) was added to the trials run in simulation to compensate for error in the real world. A global

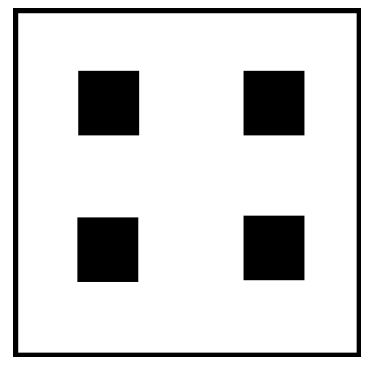

Fig. 3. The environment for simulations was $16 \times 16$ meters with four $4 \times 4$ meter obstacles.

positioning sensor (GPS) was used for localization and a laser range finder for mapping. The controller was written in the $\mathrm{C}$ programming language and experiments were performed on a Dual Core $2.33 \mathrm{GHz}$ machine running Linux with $2 \mathrm{~GB}$ of RAM. An emitter and receiver were added to each robot for point-to-point communications.

We measured the performance of each approach by examining the percentage of coverage and amount of time taken to cover an area. The No CoMm approach serves as the baseline experiment for comparison against the other approaches. The goal of the experiments was to determine whether communicating only during rendezvous is a good alternative to continuous message passing. The environment used to run the simulations was $16 \times 16$ meters with four $4 \times 4$ meter obstacles representing a city block (Figure 3 ). Twenty trials for each approach were conducted with a three-robot team.

The best approach for SECTOR REND and the ROLEBASED approach were simulated in MRESim, a JAVA-based simulation environment [20]. Currently, MRESim assumes perfect sensor data and localization. However, the simulator is used for the purpose of comparison to previous work. Each approach was simulated in the same environment shown in Figure 3. Three trials for each approach were conducted with a three-robot team.

\section{Simulation Results}

Data collected include the average time that the team required to explore at least $50 \%$ and $90 \%$ of the environment for each approach. Data also includes all averages of coverage over time.

Figure 4 illustrates the comparison between robots using No Comm, Full Comm and Prox Comm. Inspecting the figure, Full COMM and PROX COMM have similar performance. However, Full COMM covers more area at first and PROX COMM covers more at the end of exploration. On average, PROX COMM reaches $90 \%$ almost one minute faster than Full Comm (Table I).

Figure 5 illustrates the sector search with rendezvous algorithms. Overall, when compared with no communications they performed better. However, there is a point in the beginning of execution where the robots using communications covered more than those using Sector ReND 1 and SeCtOR REND 2. In Table 1, it shows that at $50 \%$ coverage, no communications is outperforming by more than two minutes. In addition, 


\begin{tabular}{|l|l|l|l|l|}
\hline & $\begin{array}{l}50 \% \text { Cover } \\
\text { Time }(\mathrm{m})\end{array}$ & $\sigma$ & $\begin{array}{l}\text { 90\% Cover } \\
\text { Time }(\mathrm{m})\end{array}$ & $\sigma$ \\
\hline No Comm & 6.63 & 1.42 & 20.97 & 3.83 \\
\hline Full Comm & 4.71 & 0.44 & 16.98 & 3.17 \\
\hline Prox Comm & 5.35 & 0.63 & 15.92 & 3.32 \\
\hline Sector Rend 1 & 7.04 & 0.33 & 15.37 & 3.47 \\
\hline Sector Rend 2 & 7.06 & 0.31 & 14.95 & 2.17 \\
\hline
\end{tabular}

TABLE I

AVERAGES FOR 50\% AND 90\% COVERAGE FOR EACH APPROACH.

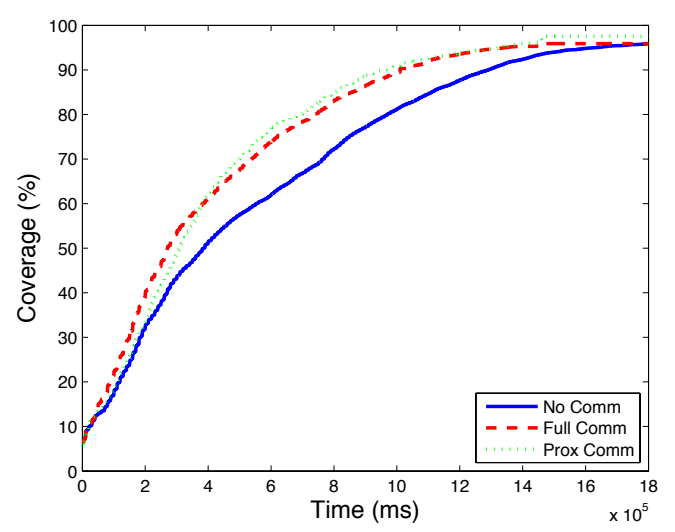

Fig. 4. A comparison of coverage and time for a robot team with no communications, direct communications during an entire exploration, and direct communications only when they are within close proximity of another robot.

SECTOR REND 1 and SECTOR REND 2 are very similar in performance to each other until about $90 \%$ at which then SECTOR REND 2 had better performance.

As shown in Figure 6, SECTOR REND 2 and PROX COMM have comparable results. SECTOR REND 2 on average covered $90 \%$ of the area quicker than PROX COMM (Table 1). However, PROX COMM demonstrated better coverage time between $30 \%$ and $70 \%$ (Figure 6).

Table I shows the average times for when at least $50 \%$ and $90 \%$ of the area was covered by the three-robot team. On average, FULL COMM reached 50\% coverage the faster. However, SECTOR REND 2 approached 90\% quicker. Figure 6 shows PROX COMM and SECTOR REND 2 performed similarly after $90 \%$.

Finally, figure 7 illustrates coverage and time for three runs each of the SECTOR REND and the ROLE-BASED approaches. While Role-based exploration outperforms sector-based exploration in the early stages, the advantages of sector based exploration soon become evident as its coverage improves. This confirms that sector-based exploration is a better solution for open, outdoor environments (whereas role-based exploration typically has the greatest advantages in communicationlimited, narrow and obstacle filled environments).

\section{ANALYSIS}

Results suggest that the alternative of having robots only communicate when in close proximity PROX COMM with each other performs better than communicating during the entire

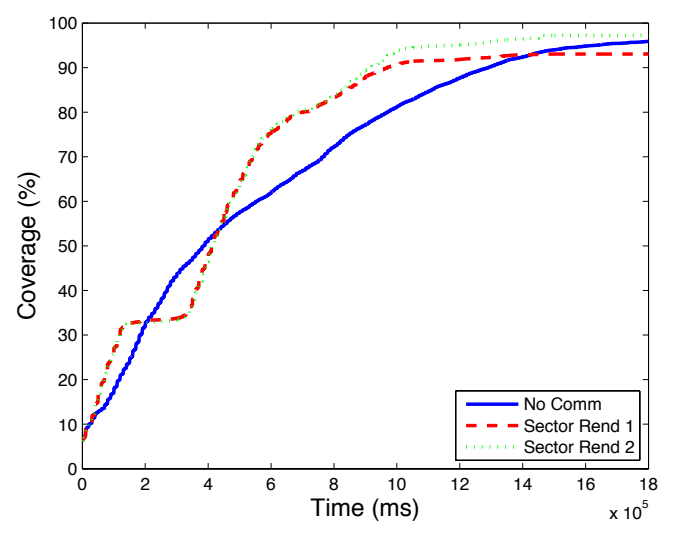

Fig. 5. A comparison of coverage and time for a robot team with no communications and algorithms of the sector search with rendezvous.

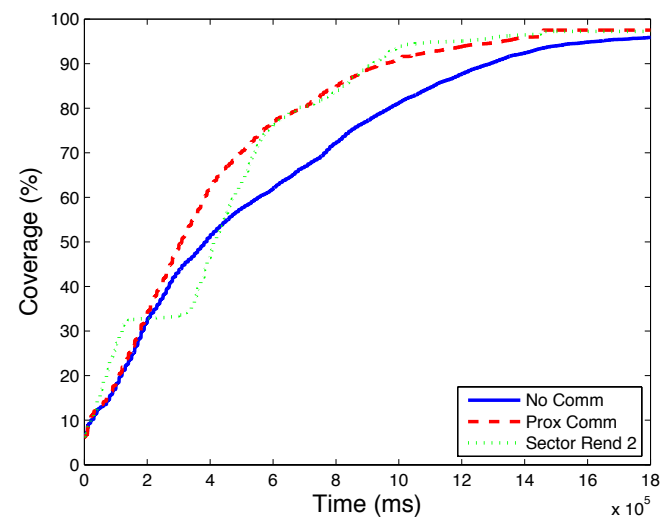

Fig. 6. A comparison of coverage and time for a robot team with no communications, direct communications only when they are within close proximity of another robot, and sector search with rendezvous approach.

exploration (Full COMM). With Full COMM, robots have more messages to process allowing for less time to explore. It also demonstrates how information that is close in both time and space is useful in immediate action selection. Additional research discusses the impact of spatial and temporal locality in information exchanged [21].

SECTOR REND 1 and SECTOR REND 2 had comparable performance but show a decrease in performance to the point where it was under no communications during the beginning of execution (Figure 5). It illustrates how taking the time to rendezvous can affect coverage. Instead of exploring, robots are meeting to exchange information. In addition, SECTOR REND 2 decrease towards the end of execution due to the amount of communications and longer rendezvous duration.

Although Prox COMM and SECTOR REND 2 had the best performance (Figure 6), they both have advantages and disadvantages. When robots only communicate when they are in close proximity with each other, they do not have to waste time to rendezvous. However, in wide open environments with small teams, there is a chance that they may not ever be in close distance of each other. In wide open environments, rendezvous 


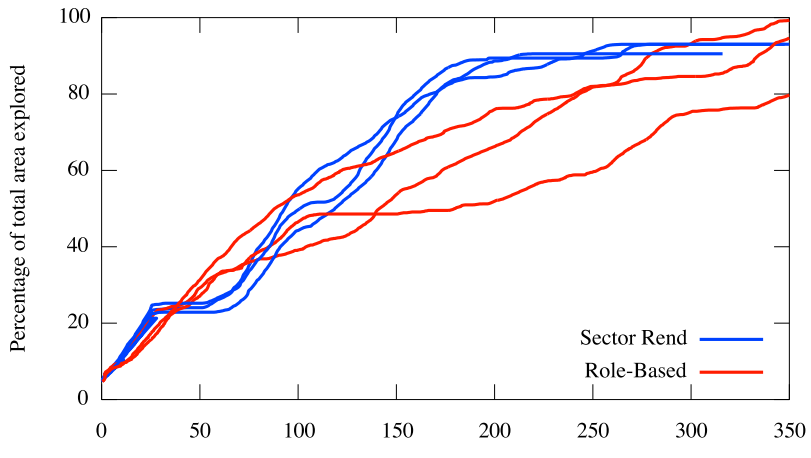

Fig. 7. Coverage and time for Role-based and Sector Search Rendezvous.

is beneficial because robots make an effort to meet instead of relying on the chance they may come in close proximity of other robots.

The utilization of rendezvous to overcome communication limitations is illustrated in both the SECTOR REND and the ROLE-BASED approaches. They are similar because they use rendezvous but task assignment is different. For example, in ROLE-BASED, robots are heterogeneous and assigned the specific roles of Explorers and Relays. In SECTOR REND, robots are homogeneous and are assigned designated areas to search. The combination of sector search and rendezvous is beneficially unique. Although sector search could be implemented without rendezvous, it offers another way to coordinate robots by preventing additional interference and duplication.

\section{CONCLUSIONS AND FutURE WORK}

An approach that uses sector search with rendezvous to coordinate robots in an exploration task of a unknown large open environment is presented. Our approach is compared to other communication paradigms in simulation. Results suggest that sector search with rendezvous leads to gains over approaches having no communications, is comparable to when robots communicate only with other robots in close proximity, and outperforms role-based exploration in open environments.

Future work includes examining sector search with rendezvous with different robot team sizes and environments. In addition, experiments with real robots will also be conducted and examining the algorithm using different rendezvous parameters is planned.

\section{ACKNOWLEDGMENT}

The authors gratefully acknowledge the support of the following NSF grants: IIS-0846976 and CCF-0829827.

\section{REFERENCES}

[1] S. Dawson, B. Wellman, and M. Anderson, "Using simulation to predict multi-robot performance on coverage tasks," in Intelligent Robots and Systems (IROS), 2010 IEEE/RSJ International Conference on. IEEE, pp. 202-208.

[2] A. J. Goldsmith and S. B. Wicker, "Design challenges for energyconstrained ad hoc wireless networks," Wireless Communications, IEEE, vol. 9, no. 4, p. 827, 2002.
[3] P. E. Rybski, S. A. Stoeter, M. Gini, D. F. Hougen, and N. Papanikolopoulos, "Effects of limited bandwidth communications channels on the controlof multiple robots," in 2001 IEEE/RSJ International Conference on Intelligent Robots and Systems, 2001. Proceedings, vol. 1, 2001.

[4] A. Howard, M. J. Mataric, and G. S. Sukhatme, "Mobile sensor network deployment using potential fields: A distributed, scalable solution to the area coverage problem," Distributed autonomous robotic systems, vol. 5, pp. 299-308, 2002.

[5] A. Howard, M. J. Matari, and G. S. Sukhatme, "An incremental selfdeployment algorithm for mobile sensor networks," Autonomous Robots, vol. 13, no. 2, pp. 113-126, 2002.

[6] S. Poduri and G. Sukhatme, "Constrained coverage for mobile sensor networks," in IEEE International Conference on Robotics and Automation, vol. 1, 2004, pp. 165-171.

[7] T. Kazama, K. Sugawara, and T. Watanabe, "Collecting behavior of interacting robots with virtual pheromone," in Distributed Autonomous Robotic Systems 6, 2007, pp. 347-356.

[8] S. Koenig, B. Szymanski, and Y. Liu, "Efficient and inefficient ant coverage methods," Annals of Mathematics and Artificial Intelligence, vol. 31, no. 1, pp. 41-76, 2001.

[9] R. C. Arkin and J. Diaz, "Line-of-sight constrained exploration for reactive multiagent robotic teams," in AMC 7th International Workshop on Advanced Motion Control, 2002, pp. 455-461.

[10] I. Rekleitis, V. Lee-Shue, A. P. New, and H. Choset, "Limited communication, multi-robot team based coverage," in IEEE International Conference on Robotics and Automation, vol. 4, 2004, pp. 3462-3468.

[11] D. Meier, C. Stachniss, and W. Burgard, "Coordinating multiple robots during exploration under communication with limited bandwidth," in ECMR, 2005, pp. 26-31.

[12] N. Roy and G. Dudek, "Collaborative robot exploration and rendezvous: Algorithms, performance bounds and observations," Autonomous Robots, vol. 11, no. 2, pp. 117-136, 2001.

[13] K. S. Senthilkumar and K. K. Bharadwaj, "A hybrid evolutionary approach for multi robot path exploration problem," in Proceedings of the International MultiConference of Engineers and Computer Scientists, vol. 2, 2008.

[14] J. de Hoog, S. Cameron, and A. Visser, "Selection of rendezvous points for multi-robot exploration in dynamic environments," in International Conference on Autonomous Agents and Multi-Agent Systems (AAMAS), 2010.

[15] — - "Dynamic team hierarchies in communication-limited multi-robot exploration," in IEEE International Workshop on Safety, Security, and Rescue Robotics (SSRR), July 2010

[16] T. Balch and R. C. Arkin, "Communication in reactive multiagent robotic systems," Autonomous Robots, vol. 1, no. 1, pp. 27-52, 1994.

[17] B. Yamauchi, "A frontier-based approach for autonomous exploration," in Proceedings of the 1997 IEEE International Symposium on Computational Intelligence in Robotics and Automation, 1997, pp. 146-151.

[18] A. Elfes, "Using occupancy grids for mobile robot perception and navigation," Computer, vol. 22, no. 6, pp. 46-57, 1989.

[19] O. Michel, "WebotsTM: professional mobile robot simulation," Arxiv preprint cs/0412052, 2004

[20] J. de Hoog, S. Cameron, and A. Visser, "Role-based autonomous multirobot exploration," in International Conference on Advanced Cognitive Technologies and Applications (COGNITIVE), November 2009.

[21] B. Wellman, S. Dawson, and M. Anderson, "Observation-based Cooperation: Pruning and Backtracking to Improve Multi-robot Coverage Performance," in Intelligent Robots and Systems (IROS), 2011 IEEE/RSJ International Conference on. IEEE, submitted. 